\title{
An Efficient Data Segmentation and Replication Technique for Cloud using Fuzzy Centrality Measures
}

\author{
S.Periyanatchi, K.Chitra
}

\begin{abstract}
Cloud computing is a creating worldview to give reliable and resilient infrastructure permitting the clients (data proprietors) to store their data and the data purchasers (clients) can get to the data from cloud servers. This worldview decreases storage and maintenance cost of the data proprietor. Notwithstanding, cloud data storage still offers ascend to security related issues. In the event of shared data, the data face both cloud-explicit and insider threats. In this work, we propose fuzzy centrality measure based division and replication of data in the cloud for perfect execution and security that consider both security and execution issues. In our framework, we separate a data records and imitate the isolated data over the cloud center points utilizing fuzzy centrality measures. Every one of the nodes stores just a solitary data fragment of a particular data document that guarantees that even if there should arise an occurrence of a fruitful attack, no significant information is uncovered to the attacker. In addition, the cloud nodes storing the data fragments, are separated with certain distance by methods for altered fuzzy T-coloring to prohibit an attacker of predicting the locations of the fragments. We likewise contrast the exhibition of the our methodology and other standard replication plans. The greater amount of security with improved performance was observed.
\end{abstract}

\section{INTRODUCTION}

Cloud handling is rapidly creating a direct result of the provisioning of flexible, versatile, and on-request storage and computing administrations for customers [1] .Organizations with a low budgetary arrangement would now be able to use high computing and storage administrations without vigorously putting resources into framework and upkeep [2] [3]. Regardless, the loss of command over information and calculation raises various security stresses for affiliations, discouraging the wide flexibility of the open cloud.

CryptoCHARTy is used as a standard technique to give secrecy and security administrations to the information [5]. The information are typically scrambled with keys before putting away to the cloud. The key administration, get to control, encryption, and decryption procedures are dealt with by the clients to guarantee information safety [6]. In any case, when the data are to be shared among a gathering, the cryptoCHARTic organizations ought to be adequately versatile to manage different customers, practice the passage control, and manage the keys in a reasonable manner to

Revised Manuscript Received on July 10, 2019.

S.Periyanatchi, Research scholar Bharathiyar university,Coimbatore, T.N, India. (Email : periyanatchi@yahoo.com) T.N, India secure information privacy [7].

To keep up a cloud to be secure, the majority of the taking an interest people must be secure. In some random cloud framework with various units, the most elevated amount of the framework security is comparable to the security level of the weakest element [8]. In this manner, in a cloud, the security of the framework does not only rely upon a person's security activities [9]. The contiguous elements may give an opportunity to an aggressor to sidestep the clients guards.

A cloud must ensure throughput, quality, and security [15]. A fundamental factor deciding the throughput of a cloud that stores information is the information recovery time[10]. In enormous scale frameworks, the challenges of information dependability, information accessibility, and reaction time are managed information replication approaches [3]. In any case, utilizing imitations information over various hubs expands the assault for that specific documents. For example, putting away $\mathrm{m}$ imitations of an information in a cloud rather than one reproduction expands the likelihood of a node holding information to be picked as assault injured individual, from $1 / \mathrm{n}$ to $\mathrm{m} / \mathrm{n}$, where $\mathrm{n}$ is the complete number of nodes

From the abovementioned, we can infer that both security and execution are basic factor for the next generation wide scale frameworks, for example, clouds. Along these lines, In this paper, we consider both security and execution issues in secure information replication issue. We present fuzzy centrality measure based discontinuity and replication of information in the cloud for ideal execution and security that gap client records into pieces and recreates them at arranged locations within the cloud. The division of a document into pieces is performed dependent on a client oblige to such an extent that the individual pieces don't contain any important information.A compelling assault on a solitary node must not uncover the areas of different records inside the cloud.

To hold an aggressor dubious about the areas of the information parts and to further improve the security, we select the nodes utilizing adjusted fuzzy $\mathrm{T}$ coloring .T shading guarantee that hubs are not neighboring and are at sure separation from one another [11]. To improve information recovery time, the hubs are chosen dependent on the fuzzy based centrality estimates that guarantee an improved access time.

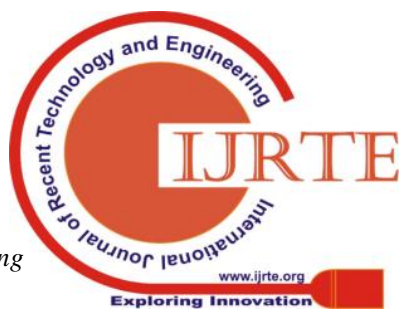




\section{AN EFFICIENT DATA SEGMENTATION AND REPLICATION TECHNIQUE FOR CLOUD USING FUZZY CENTRALITY MEASURES}

\section{RELATED WORK}

Mazhar Ali et al[12] propose the secure data sharing in clouds (SeDaSC) approach to give information classification ,access control, information sharing re encryption, insider risk security; and forward and in reverse access Control in cloud condition. The presentation of the their procedure was assessed dependent on the time utilization during the key ceation, document download, and record transfer activities. S. Website optimization et al [13] propose a certificateless encryption conspire without without key pairing for safely sharing importan data in open clouds. It tackles certificate revocation issue and key escrow issue in identity based encryption. Juels et al. [14] present a framework that offers occupants perceivability into the right procedure of the cloud. These procedures empower an expansion of as far as possible from big business interior server farms into open clouds $\mathrm{N}$. Khan et al. [15] present a dynamic accreditations based security strategy for portable client that technique offloads thr much of the time happening dynamic qualifications age and keep least calculation trouble on confided in element . T. Loukopoulos et al. [16] present a Genetic Procedure (GA) based information copy system to handle persistently evolving read/compose requests . A. Mei, et al. [17][13] present an procedure for record allotment.. Del Pozo et al [18] depict a group of centrality measures for informal organizations from a game hypothetical perspective. Get a portrayal of the centrality measures and an added substance disintegration in three summands that can be determined regarding discharge, betweenness and gathering centrality segments. In [15][16][17] researchers tried a fuzzy methodology for taking care of security issues. The data is expressed by 2-tuples, which are made out of a both semantic and numeric worth surveyed in $(-0.5,0.5)$.

\section{PRELIMINARIES}

In this section we present a few primers that we will use in the remainder of this journal

\subsection{Data Fragmentation}

The security of a cloud depends upon the security of the structure with everything taken into account and the security of autonomous hubs. An amazing interference into a solitary hub may have extraordinary disciplines, not only for data and applications on the injured individual hub, yet furthermore for different hubs. The data on the harmed individual hub may be revealed totally in light of the closeness of the entire record [17].

The quantity of compromised data can be decreased by making pieces of an information document and putting away them on different nodes [17].A effective interference on a single node or few nodes will just give access to a segment of information that probably won't be of any helpful in information. Additionally, if an assailant is dubious about the areas of the information sections, the likelihood of discovering information pieces on the majority of the nodes is exceptionally low

\subsubsection{DCM-Degree Centrality Measure}

The DC characterized as local CM since it is calculated by just its directed associations. The DC of a node is determined by including of its approaching (id-indegree) and active (od-outdegree) association loads:

\section{$\mathrm{CD}($ node $)=\sum($ id $($ node $)+$ od $($ node $))$}

where the id(v) is the addition of association loads entering hub $\mathrm{v}$, and the od(v) is the addition of association loads leaving hub $\mathrm{v}$

\subsubsection{BCM-Betweenness Centrality Measure}

The $\mathrm{BC}$ characterized as worldwide $\mathrm{CM}$ since it is determined dependent on the briefest ways between hub combines in the CHART. The BC of a node is identified by including the extent of most limited ways between nodes combines that experience that nodes .for the directed CHART $\mathrm{G}=(\mathrm{V}, \mathrm{E})$, the $\mathrm{BC}$ of a node $\mathrm{v}$ is characterized as:

$$
\mathrm{C}_{\mathrm{B}}=\sum_{S \pm v \pm t \in V} \sigma s t(\mathrm{v}) / \sigma s t
$$

Where $\sigma$ st speaks to number of most limited ways from node $s$ to node $t$ and $\sigma s t(v)$ is the quantity of briefest ways from $\mathrm{s}$ to $\mathrm{t}$ that goes through node $\mathrm{v}$.

\subsubsection{CCM-Closeness Centrality Measure}

Like betweenness, the CCM is a global CM determined dependent on the most limited ways idea. It discovers how close a node is near every single other node in the chart. For a directed CHART $G=(V, E)$, the closeness centrality of a node $\mathrm{v}$ is characterized as:

$$
\mathrm{Cc}(\mathrm{v})=\frac{1}{\sum t \in V d_{G(\mathrm{v}, t)}}
$$

Where $\mathrm{t} \neq \mathrm{v}$, and $\mathrm{dG}(\mathrm{v}, \mathrm{t})$ is the most brief way between nodes $v$ and $t$. As to cloud network, the CCM is a proportion of how rapidly a node speaks with different nodes in the cloud network

\subsubsection{A 2-Tuple (FLM) Fuzzy Linguistic Representation} Model

A 2-tuple FLM was created based on an emblematic linguistic model. Let $S=\{\mathrm{s} 0, \ldots, \mathrm{sg}\}$ be a linguistic term set, for example, low, medium, and high and so forth., where si < $\mathrm{sj}$ if and just in the event that $\mathrm{I}<\mathrm{j}$ and $\mathrm{g}+1$ is the quantity of linguistic terms. The emblematic model indicates the linguistic data by an arranged linguistic term set like $\mathrm{S}$ and utilizations the arranged list I, I $\in[0, \mathrm{~g}]$, of the linguistic term set $\mathrm{S}$ to play out the figurings.

In the 2-tuple, the (LT) linguistic term is signified by a 2 -tuple (s, $\alpha$ ) where s speaks to LT and $\alpha \in[-0.5,0.5)$ is an emblematic interpretation. In light of the abovementioned, the 2-tuple model speaks to the linguistic data in consistent estimate process thus, it handles any including of numbers in data coming about because of representative total

To explain the 2-tuple model, let $\mathrm{S}=\{\mathrm{s} 0, \ldots, \mathrm{s} 6\}$ be an emblematic LT set where each LT si is indicated by triangular parameters (ai, bi, ci) and $\mathrm{I}=0, . ., \mathrm{g}$ is the file of si, and $\mu \mathrm{si}(\mathrm{n})$ is participation capacity esteems related with si for a particular worth (n) of a $\mathrm{CM}$ and determined

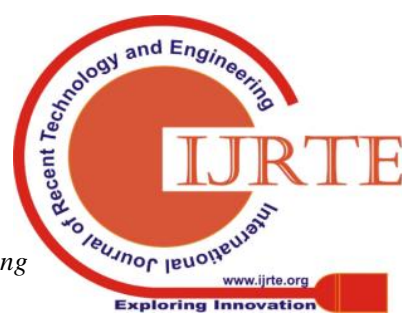


utilizing the accompanying Equation:

$$
\mu_{s_{i}}(n)=\left\{\begin{aligned}
\frac{n-a_{i}}{b_{i}-a_{i}}, & a \leq n \leq b \\
\frac{c_{i}-n}{c_{i}-b_{i}}, & b<n \leq c \\
0, & \text { otherwise }
\end{aligned}\right.
$$

Let $\beta \epsilon[0, \mathrm{~g}]$ be the outcome of a weighted aggregation of the labelled indices in the LT set S

\section{Our proposed Model}

Consider a cloud that contains $\mathrm{N}$ number of nodes, each with its own putting away limit. Let $\mathrm{CNi}$ means the name of I-th node and cni indicates complete storage limit of $\mathrm{CNi}$. The correspondence time among $\mathrm{CNi}$ and $\mathrm{CNj}$ is the complete time of the majority of the connections inside a chose way from $\mathrm{CNi}$ to $\mathrm{CNj}$ spoken to by $\operatorname{com}(\mathrm{i}, \mathrm{j})$. We consider $\mathrm{N}$ of information sections to such an extent that $\mathrm{Fk}$ signifies $\mathrm{k}$-th part of a record while fk speaks to the size of $\mathrm{k}$-th piece. Let the complete read and compose demands from $\mathrm{CNi}$ for Fk be spoken to by reik and wrik, separately. Let Prk mean the essential node that stores the essential duplicate of Fk. The replication conspire for Fk meant by Rek is likewise put away at Prk. Also, every CNi comprise of a two-field record, putting away Prk for Fk and NNi k that speaks to the closest node putting away Fk. At whatever point there is an update in Fk, the invigorated interpretation is sent to Prk that spreads the revived variation to most of the hubs in Rek. Let bw $(i, j)$ and $\operatorname{tr}(i, j)$ be the total information transmission of the association and traffic between goals $\mathrm{CNi}$ and $\mathrm{CNj}$, independently. The $\mathrm{CM}$ for $\mathrm{CNi}$ is addressed by centi . Let colorSi store the estimation of dispensed concealing to $\mathrm{Si}$. The colorSi can have one out of two characteristics, specifically: open concealing and close concealing. The value open concealing means that the hub is available for securing the report segment. The value close concealing shows that the hub can't store the record part. Let INT be a ton of entire numbers starting from zero and conclusion on a pre-portrayed number. If the picked number is three, by then INT $=\{0 ; 1 ; 2 ; 3\}$. The set INT is used to oblige the center point decision to those hubs that are at skip partitions not having a spot with INTOur point is to diminish the overal information moving time or replication time (ReT) or in like manner named as replication cost $(\mathrm{ReC})$. The ReT is made out of two factors: (a) Time for read requesting and (b) time for make requests. The hard and fast perused time of $\mathrm{Fk}$ by

$$
\operatorname{Re}_{\mathrm{k}}^{\mathrm{i}}=\operatorname{re}_{\mathrm{k}}^{\mathrm{i}} \mathrm{F}_{\mathrm{k}} \operatorname{com}\left(\mathrm{i} ; \mathrm{NN}^{\mathrm{i}}{ }_{\mathrm{k}}\right)
$$
$\mathrm{CNi}$ from NNi $\mathrm{k}$ is implied by Reik and is given by:

The total time due to the writing of $\mathrm{O}_{\mathrm{k}}$ by $\mathrm{S}^{\mathrm{i}}$ addressed to the $\operatorname{Pr}_{\mathrm{k}}$ is represented as $\mathrm{Wr}_{\mathrm{k}}^{\mathrm{i}}$ and is given:

$\mathrm{Wr}_{\mathrm{k}}^{\mathrm{i}}=\mathrm{wr}_{\mathrm{k}}^{\mathrm{i}} \mathrm{F}_{\mathrm{k}}\left(\operatorname{com}\left(\mathrm{i} ; \operatorname{Pr}_{\mathrm{k}}\right)+\sum(j \in R e k)_{j}{ }^{n} \quad \operatorname{com}\left(\operatorname{Pr}_{\mathrm{k}} ; \mathrm{j}\right)\right)$ (6)

The overall RT is represented by:

$$
\operatorname{ReT}=\sum_{I=1}^{M} \quad \sum_{k=1}^{N} \quad\left(\mathrm{R}_{\mathrm{k}}^{\mathrm{i}}+\mathrm{W}_{\mathrm{k}}^{\mathrm{i}}\right)
$$

The storage capacity limitation states that a file fragment can only be assigned to a node, if storing capacity of the node is greater or equal to the size of file fragment.

\section{PROPOSED METHOD}

The principle target of this work is to acquire a fuzzy based $\mathrm{CM}$ from three previously mentioned CM for recognizing the focal nodes and afterward organizing them as per their centrality significance. So as to find that, we calculate CM of each nodes independently. Besides, we change numeric $\mathrm{CM}$ into emblematic FLT, and afterward we utilize the 2-tuple FLM to change over the LT into 2-tuple FLM set. Thirdly, we utilize the 2-tuple combination system and number juggling mean administrator to join the 2-tuple FLM of the measures. Subsequently, joined estimation of the hub centrality count indicates a hub's significance in correlation with different hubs, i.e., the bigger worth the hub centrality worth is the higher the nodes's significance. At last, we rank the nodes in sliding request as per their centrality significance. We can utilize this rundown to improve arranges by expelling the least significant nodes dependent on an edge that characterizes the quantity of required nodes in system. Also, these centrality estimations of the nodes give a sign of the most significant nodes that helps the chief in investigating the structure of the cloud nodes and deciding.

To ascertain the DC of a node, we speak to a nearness matrix $\mathrm{E}$ of measurement $\mathrm{N} x \mathrm{~N}$, where $\mathrm{N}$ is the quantity of hubs in the cloud and every component in the matrix ei,j speaks to the association weight from hub I to hub $\mathrm{j}$. At that point, we figure the indegree id and outdegree od for every hub utilizing the Equations 8 , individually: Then, we apply Equation 1 to ascertain the DC for all hubs in cloud .

$$
\begin{aligned}
& i d(i)=\sum_{j=1}^{N}\left|e_{j i}\right| \\
& \operatorname{od}(i)=\sum_{j=1}^{N}\left|e_{i j}\right|
\end{aligned}
$$

\section{Modified fuzzy T coloring}

Channel task $(\mathrm{CH})$ issue characterized by Hale[24], the $\mathrm{T}$-shading upgrade issue for channel task $\left(\mathrm{F}^{*} \mathrm{D}\right.$ obliged cochannel assignment issue (F*D-CCAP)) allocates channels to the hubs, to such a degree, that the $\mathrm{CH}$ 's are confined by a some

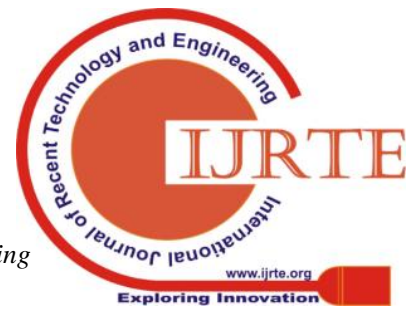




\section{AN EFFICIENT DATA SEGMENTATION AND REPLICATION TECHNIQUE FOR CLOUD USING FUZZY CENTRALITY MEASURES}

detachment to keep up a vital separation from impedance. For a $\mathrm{CHART} \mathrm{G}=(\mathrm{V} ; \mathrm{E})$ and a set $\mathrm{T}$ containing non-negative entire numbers including 0 . Our fuzzy $T$ shading is a mapping limit $\mathrm{f}$ from the vertices of $\mathrm{V}$ to the course of action of non-negative numbers, with the true objective that function(a)- function(b), where $(a ; b)>E$. The fuzzy support changing over limit $\mathrm{f}$ apportions a concealing to a vertex subject to security level. $\mathrm{CH}$ errand consigns fuzzy $\mathrm{CH}$ to the hubs, to such a degree, that the $\mathrm{CH}^{\prime}$ s are confined by a partition to keep up a vital separation from security level issues .

In the our system, we propose not to store the whole information document at a solitary hub. The part and replication system sections the record and utilizes the cloud for information replication. The parts are spread with the ultimate objective that no hub in a cloud holds more than a solitary section, so that even an incredible strike on the hub discharges no basic information. our replication strategy uses controlled replication where every one of the information sections is recreated just once in the cloud to improve the security. In spite of the fact that, the controlled information replication does not improve the recovery time to the degree of full-scale replication, it altogether improves the security.

Our proposed work as pursues, client sends the information record to cloud. The cloud administrator framework after accepting the document plays out: (a) fragmentation, (b) fuzzy centrality based nodes choice and stores one part over every one of the selected node, and (c) adjusted fuzzy $\mathrm{T}$ coloring node selection for sections replication. The cloud administrator keeps up record of the part position and is thought to be a safe element.

When the document is separated into fragments, Proposed technique chooses the cloud node for fragment placement . The determination is finished by keeping an equivalent consideration on both security and execution regarding the access time. We pick the nodes that are most integral to the cloud system to offer better access time. Consequently, our technique uses the possibility of fuzzy centrality to reduce access time. The centralities choose how central a center point relies upon different measures as discussed in section3.2. We select hub with three CM's, to be specific: (a) DCM , (b) BCM, and (c) CCM . In any case, if the majority of the information sections are put on the nodes dependent on the sliding request of centrality, at that point quite possibly contiguous nodes are chosen for part situation.

Such a position can give indications to an aggressor regarding where different fragments may be available, lessening the security level of the information. To manage the security parts of setting information sections, we utilize the idea of changed fuzzy T-coloring. For the said reason, we distribute colors to the nodes, to such an extent that, at first, the majority of the nodes are given the open colring. When an information section is set on the node, the majority of the nodes inside the area at a separation having a place with $\mathrm{T}$ are assigned close coloring. The procedure is proceeded until the majority of the information pieces are put at the nodes . Procedure 1 explains the piece or fragment position approach

\section{Procedure 1}

Procedure for data fragment placement Inputs and initializations:

$\mathrm{F}=\{\mathrm{F} 1 ; \mathrm{F} 2 ;:: ; ; \mathrm{FN}\}$

$\mathrm{f}=\{$ size of $(\mathrm{F} 1)$; size of $(\mathrm{F} 2) ;:: .: ;$ size of $(\mathrm{FN})\}$

color $=\{$ open color; close color $\}$

cent $=\{$ cent $1 ;$ cent $2 ;::: ;$ cent $\mathrm{M}\}$

color $<$ - open color $\vee \mathrm{i}$

cent $<$ - centi $\vee \mathrm{i}$

Compute:

for each $\mathrm{F}_{\mathrm{k}} \in \mathrm{F}$ do

select $\mathrm{CN}^{\mathrm{i}} \mid \mathrm{CN}^{\mathrm{i}} \leftarrow \operatorname{indexof}(\max ($ centi) $)$

if color $_{\mathrm{CN}} \mathrm{i}=$ open color and $\mathrm{cni}>=\mathrm{f}_{\mathrm{k}}$ then

$\mathrm{CN}^{\mathrm{i}}<-\mathrm{F}_{\mathrm{k}}$

$\mathrm{cn}_{\mathrm{i}}<-\mathrm{cni}-\mathrm{fk}$

color $_{\text {cni }}<$-close color

$\mathrm{CN}^{\mathrm{i}}<$-distance $\left(\mathrm{CN}^{\mathrm{i}}\right.$; T) $\mathrm{P} /$ *returns all nodes at

distance $\mathrm{T}$ from $\mathrm{Si}$ and stores in temporary set $\mathrm{Si}^{\prime}{ }^{*} /$

col $_{\text {cni. }}$ <- close color

end if

end for

\section{Procedure 2}

Procedure for data fragment replication

for each $\mathrm{F}_{\mathrm{k}}$ in $\mathrm{F}$ do

select $\mathrm{CNi}$ that has $\max \left(\operatorname{Re}_{\mathrm{k}}^{\mathrm{i}}+\mathrm{Wr}_{\mathrm{k}}^{\mathrm{i}}\right)$

if color $_{\mathrm{cni}}=$ open color and $\mathrm{cni}>=\mathrm{fk}$ then

$\mathrm{CN}^{\mathrm{i}}<-\mathrm{F}_{\mathrm{k}}$

cni $<-$ cni - fk

color $_{\text {cni }}<-$ close color

$\mathrm{CNi}$ '<-distance $(\mathrm{CNi}$; $\mathrm{T}) \mathrm{P} / *$ returns all nodes at

distance $\mathrm{T}$ from $\mathrm{Si}$ and stores in temporary set $\mathrm{Si}^{*} * /$

color $_{\text {cni. }}<$-close color

end if

end for

The information replication procedure is clarified in Procedure 2. To deal with the download demand from customer, the cloud director gathers every one of the information sections from the nodes and reassemble them into a single document. A while later, the document is sent to the customer

\section{RESULT AND DISCUSSION}

We implement and compared the results of the DROPS methodology with well- known replication strategies, namely: (a) DRPA-star, (b)SA1 (c) SA2, (d) WA-star, (e) A-star, (f) Local Min-Min, (g) SA3, (h) Global Min- Min, (i) Greedy procedure , and (j) Genetic Replication Procedure (GRA).

At whatever point there is an update in $\mathrm{Fk}$, the invigorated interpretation is sent to Prk that spreads the revived variation to most of the hubs in Rek. Let bw(i,j) and tr(i,j) be the total information transmission of the association and traffic between goals $\mathrm{CNi}$ and $\mathrm{CNj}$, independently. The $\mathrm{CM}$ for $\mathrm{CNi}$ is addressed by centi . Let colorSi store the estimation of dispensed concealing to Si. The colorSi can have one out

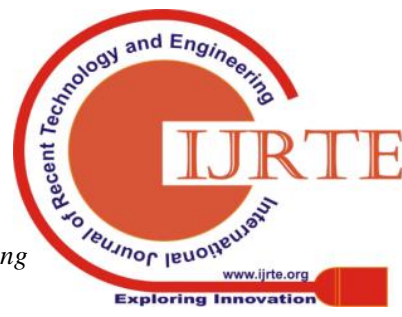


of two characteristics, specifically: open concealing and close concealing. The value open concealing means that the hub is available for securing the report segment. The value close concealing shows that the hub can't store the record part. Let INT be a ton of entire numbers starting from zero and conclusion on a pre-portrayed number. If the picked number is three, by then INT $=\{0 ; 1 ; 2 ; 3\}$. The set INT is used to oblige the center point decision to those hubs that are at skip partitions not having a spot with INT.

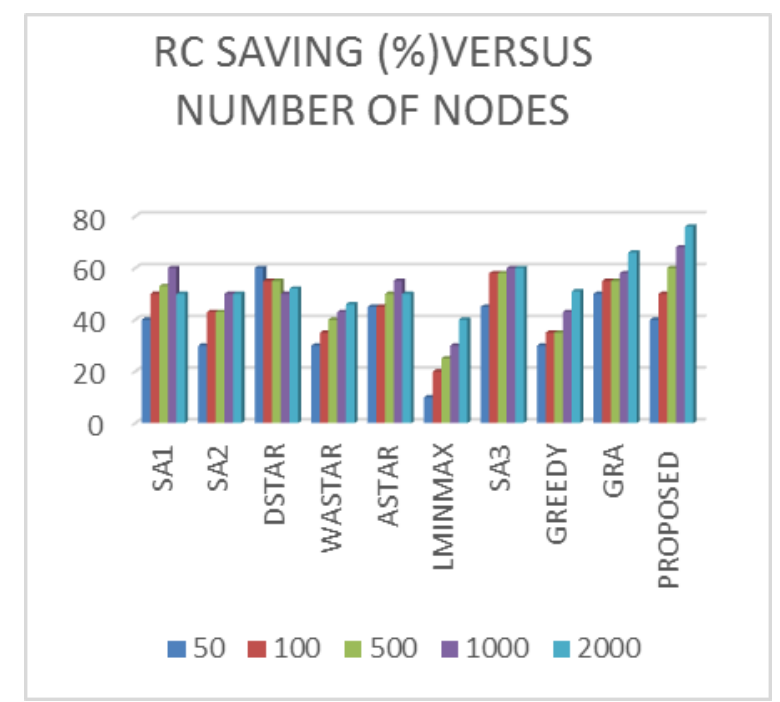

Figure 1 RC versus number of node

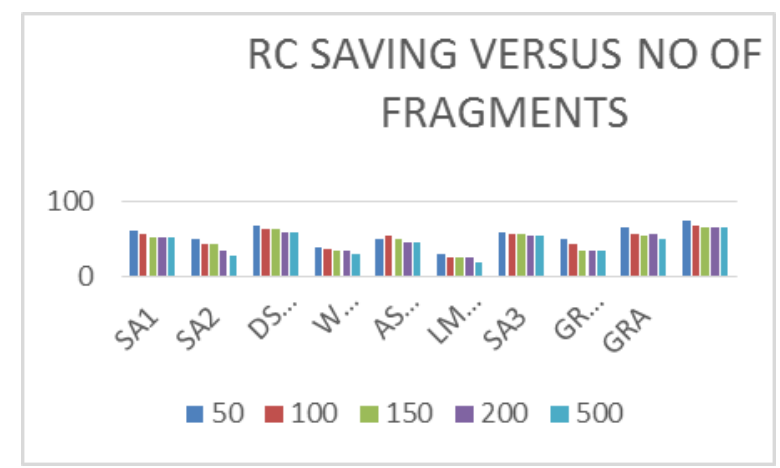

Figure 2 RC versus number of fragments

\section{CONCLUSIONS}

We proposed the fuzzy based information replication framework, consider both security level and execution to the degree recovery time. The customer information was isolated and the pieces are spread over different center points. The center points were isolated by methods for adjusted fuzzy T-shading. The information irregularity and dispersal guaranteed that no huge data was open by a foe if there should be an occasion of a strike. No middle point in the cloud, set away in excess of an information part of a relative document. The presentation of the proposed philosophy was separated and standard replication techniques. The aftereffects of the reenactments shows that the synchronous spotlight on the security and execution, accomplished expanded security level and execution improvement

\section{REFERENCES}

1. Seo et al, "A review on the State-of-the-art privacy preserving approaches in e-health clouds," IET ,

2. K. jules et al., "An overview of the commercial cloud monitoring tools: Research dimensions, design issues, state-of-the-art," Computing

3. A. N. Khan, M. L. M. Kiah, S. U. Khan, and S. A. Madani, "Towards secure mobile cloud computing: A survey," journal of FGCS

4. L. Wei, et al , "Security and privacy for storage and computation in cloud computing,", JPDC

5. Cloud security Alliance, "Security guidelines for critical areas of focus in cloud computing v3.0," 2011.

6. D. Chen et al., "Fast and scalable multi-way analysis of massive neural data," ACM Trans. Comput., vol. 64, no. 3, pp. 707-719, Mar. 2015.

7. A.N. Khan et al, "Incremental proxy re-encryption scheme for mobile cloud computing environment," ijstas.

8. R. Kaaufman, "Data security in the world of cloud computing," JSP

9. T.Walloscheket et al "Understanding cloud computing vulnerabilities," ACM journal

10. Bastani et al , "On the optimal placement of secure data objects over Internet," cloud Processing Symposium

11. W. Hale, "Frequency assignment: Theory and applications," Proceedings of the IET

12. Mazhar Ali et al : Secure Data Sharing in Clouds, ISJ , VOL. 21, NO 3, JUNE 2019

13. S. Seo et al, "An Efficient Certificateless Encryption for Secure Data Sharing in Public Clouds," ACM

14. Opera et al, "New approaches to security and availability for cloud data," IEEE

15. A. N. Khan et al, "Towards Secure Mobile Cloud Computing: A Survey," IEEE.,

16. Ahmad et al, "Static and adaptive distributed data replication using genetic procedure s," Sci Journal

17. V. Mancini et al, "Secure dynamic fragment and replica allocation in large-scale distributed file systems," IEEE 